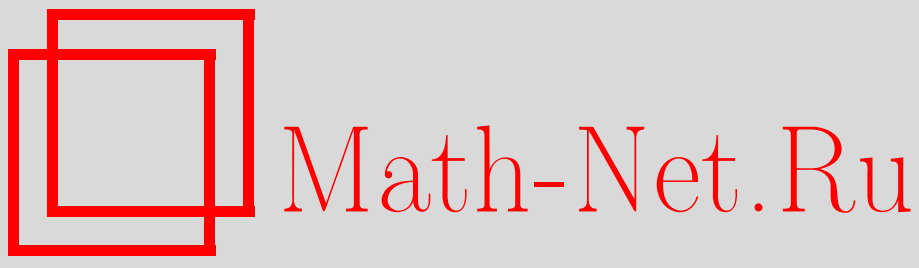

В. Л. Попов, О полиномиальных автоморфизмах аффинных пространств, Изв. РАН. Сер. матем., 2001, том 65, выпуск 3, 153-174

DOI: https://doi.org/10.4213/im340

Использование Общероссийского математического портала Math-Net.Ru подразумевает, что вы прочитали и согласны с пользовательским соглашением http: //www. mathnet.ru/rus/agreement

Параметры загрузки:

IP : 54.164 .48 .24

26 апреля 2023 г., $16: 52: 25$ 
УДК 512.745

\author{
В. Л. Попов
}

\title{
О полиномиальных автоморфизмах аффинных пространств
}

\begin{abstract}
В первой части работы доказано несколько общих результатов о линеаризуемости действий алгебраических групп на $\mathbb{A}^{n}$. В качестве приложения получен метод построения (и приведен конкретный пример) нелинеаризуемых алгебраических действий бесконечных нередуктивных неразрешимых алгебраических групп на $\mathbb{A}^{n}$, обладающих неподвижной точкой. Во второй части эти общие результаты используются для доказательства того, что всякое эффективное алгебраическое действие связной редуктивной алгебраической группы $G$ на $n$-мерном аффинном пространстве $\mathbb{A}^{n}$ над алгебраически замкнутым полем $k$ нулевой характеристики линеаризуемо в каждом из следующих случаев: 1) $n=3$;2) $n=4$ и $G$ не является одно- или двумерным тором. В частности, это означает, что $\mathrm{GL}_{3}(k)$ - единственная с точностью до сопряженности максимальная связная редуктивная подгруппа в группе автоморфизмов алгебры полиномов от трех переменных над $k$.

Библиография: 32 наименования.
\end{abstract}

\section{$\S 1$. Введение}

1.1. В дальнейшем $k$ обозначает алгебраически замкнутое поле нулевой характеристики. Если не оговорено отдельно, все алгебраические многообразия и морфизмы считаются определенными над $k$, алгебраические многообразия отождествляются с пространствами их $k$-точек и все действия считаются алгебраическими.

1.2. Мы рассматриваем в этой работе алгебраические действия редуктивных алгебраических груп $G$ на $n$-мерном аффинном пространстве $\mathbb{A}^{n}$. Известно, что если группа $G$ связна и не является тором, а $n$ достаточно велико, то существует нелинеаризуемое действие групшы $G$ на пространстве $\mathbb{A}^{n}$ (доказательство утверждений, приводимых далее без ссылок, см. в [18] и в цитированной там литературе). Однако если $n$ мало, имеются результаты положительного характера, так что в этом случае ситуация является более тонкой.

А именно, согласно классическому результату действия редуктивных алгебраических групп на пространстве $\mathbb{A}^{n}$ при $n \leqslant 2$ линеаризуемы. В работе [19] было доказано, что при $n=3$ действия связных полупростых алгебраических групп на пространстве $\mathbb{A}^{n}$ линеаризуемы. Затем в [24] было доказано, что последнее верно

Работа выполнена при поддержке CRDF (грант RM1-206).

$$
\text { (C) в. Л. Попов, } 2001
$$


и при $n=4$ (работа [24] основана на идеях и методах появившейся раньше препринтной версии работы [19]). Недавно, после почти пятнадцатилетних усилий, была доказана линеаризуемость действий одномерного тора на пространстве $\mathbb{A}^{3}$ (отдельные части этого доказательства являются результатами работ [12], [15], [14], [9], [16] и [17]; см. исторические подробности в [10]). Все сказанное свидетельствует о том, что, как и $n=1,2$, размерности $n=3,4$ являются исключительными в отношении линеаризуемости действий.

1.3. В настоящей работе получены две групшы результатов.

В первой половине работы доказывается ряд обших утверждений, относяшихся к линеаризуемости действий. Мы отсылаем читателя к $\S 2$ за формулировками. В качестве одного из приложений мы получаем метод построения и конкретный пример нелинеаризуемого действия бесконечной нередуктивной неразрешимой алгебраической группы на пространстве $\mathbb{A}^{n}$, обладающего неподвижной точкой (см. п. 2.10).

Вторая половина работы содержит приложения результатов из первой части к анализу структуры связных алгебраических подгрупп в Aut $\mathbb{A}^{3}$ и Aut $\mathbb{A}^{4}$. Мы получаем следующие результаты.

Теорема 1. Всякое алгебраическое действие связной редуктивной алгебраической группы на $\mathbb{A}^{3}$ линеаризуемо.

Теорема 2. Всякое әффективное алгебраическое действие на $\mathbb{A}^{4}$ связной редуктивной алгебраической группь, отличной от одно- или двумерного тора, линеаризуемо.

1.4. В доказательстве теоремы 1 существенно используется линеаризуемость действий одномерного тора на $\mathbb{A}^{3}$. Результаты из первой части быстро приводят к этому результату и линеаризуемости действий связных полупростых групп на $\mathbb{A}^{3}$. Напротив, в доказательстве теоремы 2 линеаризуемость действий одномерного тора на $\mathbb{A}^{3}$ не используется.

1.5. Теоремы 1 и 2 , очевидно, могут быть переформулированы следуюшим образом.

Теорема $1^{\prime}$. Любая связная редуктивная алгебраическая подгруппа в $\operatorname{Aut}^{3}$ (или, әквивалентно, в группе автоморфизмов алгебры полиномов от трех переменных над полем $k$ ) сопряжсена подгруппе группы $\mathrm{GL}_{3}(k)$.

ТЕорема $2^{\prime}$. Любая связная редуктивная алгебраическая подгруппа в $\operatorname{Aut}^{4}$ (или, әквивалентно, в группе автоморфизмов алгебры полиномов от четырех переменных над полем $k$ ), отличная от одно- или двумерного тора, сопряжсена подгруппе группы $\mathrm{GL}_{4}(k)$.

1.6. Можно показать (см. ниже следствие из теоремы 4$)$, что группа $\mathrm{GL}_{n}(k)$ является максимальной редуктивной подгруппой бесконечномерной (при $n \geqslant 2$ ) алгебраической (см. [32], [11]) группы Aut $\mathbb{A}^{n}$. В конечномерной алгебраической группе $H$ любая максимальная редуктивная подгруппа $R$ является ее подгруппой 
Леви (т.е. $H$-полупрямое произведениегруппы $R$ и унипотентного радикала группы $H$ ). Теоремы 1 и $1^{\prime}$, очевидно, эквивалентны следующему утверждению, показываюшему, что бесконечномерная алгебраическая группа Aut $\mathbb{A}^{3}$ (как и группа Aut $\mathbb{A}^{2}$ ) обладает одним из фундаментальных свойств конечномерных алгебраических групп - сопряженностью подгрупп Леви.

Teорема 3. В группе Aut $\mathbb{A}^{3}$ имеется ровно одна, с точностью до сопряженности, максимальная связная редуктивная алгебраическая подгруппа, а именно $\mathrm{GL}_{3}(k)$.

1.7. Сложность доказательства линеаризуемости действий одномерных торов на $\mathbb{A}^{3}$ свидетельствует в пользу того, что если утверждения теорем 2 и $2^{\prime}$ справедливы без предположения, что $G$ не одно- или двумерньй тор (и, значит, если имеет место аналог теоремы 3 для группы Aut $\mathbb{A}^{4}$ ), то доказать это будет нелегко. Отметим, что имеется семейство неэквивалентных нелинеаризуемых алгебраических действий ортогональной групшы $\mathrm{O}_{2}(k)$ (т.е. некоммутативного расширения одномерного тора с помощью группы второго порядка) на пространстве $\mathbb{A}^{4}$ (см. [22]). В этом смысле случай действий одномерного тора на пространстве $\mathbb{A}^{4}$ расположен "на краю" той области, где линеаризуемость действий имеет место. Случай действий двумерного тора на пространстве $\mathbb{A}^{4}$ кажется более доступным.

1.8. Ввиду сушествования нелинеаризуемых действий несвязных редуктивных алгебраических групп на пространстве $\mathbb{A}^{4}$ предположение о связности в теоремах 2 и $2^{\prime}$ не может быть снято. Насколько я знаю, остается открытым вопрос: сушествуют ли такие примеры (в частности, нелинеаризуемые действия конечных групп) при $n=3$ ? Другой открытый вопрос: исчерпывают ли случаи $n \leqslant 4$ все исключительные размерности в отношении линеаризуемости действий связных редуктивных алгебраических групп, отличных от торов? Наименьшая известная мне размерность, в которой не любое действие таких групп линеаризуемо, - это $n=7$.

1.9. В качестве основной ссылки относительно стандартных обозначений и базисных фактов теории алгебраических действий используется работа [29]. Единственное исключение: категорный фактор для действия редуктивной алгебраической группы $G$ на аффинном алгебраическом многообразии $X$ обозначается через $\pi_{X, G}: X \longrightarrow X / / G$, как теперь обшепринято.

$\operatorname{Aut}_{H} Y$ - групп $H$-эквивариантных автоморфизмов алгебраического многообразия $Y$, на котором действует алгебраическая группа $H$.

$V_{d}$-простой алгебраический $(d+1)$-мерный $\mathrm{SL}_{2}(k)$-модуль, $d \in \mathbb{N}:=\{0,1,2, \ldots\}$ (с точностью до изоморфизма такой имеется ровно один).

1.10. Я благодарен П. Расселлу, чей вопрос о справедливости теоремы 1 был для меня отправной точкой и привел к появлению препринтов [27] и [28], и Х. Бассу за полезную переписку. Я признателен за гостеприимство сотрудникам института математики Иннсбрукского университета, где была завершена настояшая работа. 


\section{§ 2. Некоторые общие результаты о действиях алгебраических групп}

2.1. В этом параграфе получено несколько общих результатов об алгебраических действиях. Во второй половине работы они будут использованы в доказательствах теорем 1 и 2.

2.2. Следуюшие три утверждения, теоремы 4-6, хорошо известны.

Теорема 4 [19]. Пусть редуктивная алгебраическая группа $G$ действует на пространстве $\mathbb{A}^{n}$. Если категорный фактор $\mathbb{A}^{n} / / G$ является точкой (т.е. $\left.k\left[\mathbb{A}^{n}\right]^{G}=k\right)$, то действие линеаризуемо.

СлЕДСТВИЕ 1. Группа $\mathrm{GL}_{n}(k)$ является максимальной редуктивной подәруппой в Aut $\mathbb{A}^{n}$.

ДоКАЗАТЕЛЬСТво. Поскольку группа $\mathrm{GL}_{n}(k)$ имеет в пространстве $\mathbb{A}^{n}$ открытую орбиту, это же верно для любой редуктивной подгрупшы $G$ в Aut $\mathbb{A}^{n}$, coдержащей группу $\mathrm{GL}_{n}(k)$. Поэтому $k\left[\mathbb{A}^{n}\right]^{G}=k$. Значит, по теореме 4 действие группы $G$ на пространстве $\mathbb{A}^{n}$ линеаризуемо, т.е. группа $G$ сопряжена в Aut $\mathbb{A}^{n}$ подгруппе групшы $\mathrm{GL}_{n}(k)$. Поскольку $\mathrm{GL}_{n}(k) \subseteq G$, это дает $G=\mathrm{GL}_{n}(k)$.

2.3. Напомним [2], что действие редуктивной алгебраической группы на аффинном алгебраическом многообразии называется fixed pointed, если лишь неподвижные точки являются замкнутыми орбитами.

Теорема 5 [2]. Пусть редуктивная алгебраическая группа $G$ действует на пространстве $\mathbb{A}^{n}$. Eсли это - fixed pointed-действие, то оно линеаризуемо тогда и только тогда, когда многообразие $\left(\mathbb{A}^{n}\right)^{G}$ неподвижных точек изоморфно пространству $\mathbb{A}^{s}$ для некоторого $s ;$ при $s \leqslant 2$ это условие выполнено автоматически.

2.4. Следующее утверждение является стандартным фактом теории однородных пространств (см., например, [8, теорема 1.2]). Его доказательство является легким упражнением.

Tеорема 6. Пусть $H$ - алгебраическая группа и $Q-$-е замкнутая подгруппа. Рассмотрим действие группь $H$ на однородном пространстве $H / Q$, определенное левыми сдвигами. Тогда группа Aut $_{H} H / Q$ совпадает с образом группь $N_{H}(Q) / Q$, действующей на пространстве $H / Q$ с помощью правых сдвигов. Это действие эффективно, так что группа $\operatorname{Aut}_{H} H / Q$ изоморфна zpynne $N_{H}(Q) / Q$.

2.5. Результаты, содержашиеся в пाп. $2.5,2.6$, касаются связи между линейностью эквивариантного автоморфизма и некоторыми свойствами разложений на изотипные компоненты.

А именно, пусть $G$ - редуктивная алгебраическая группа и $V$ - конечномерньй алгебраический $G$-модуль. Поскольку всякая изотипная компонента $G$-модуля $k[V]$ является конечно порожденным $k[V]^{G}$-модулем, некоторая (эквивалентно, 
любая) ненулевая изотипная компонента конечномерна тогда и только тогда, когда $k[V]^{G}=k$. Поскольку группа $G$ действует на пространстве $V$ линейно, $V^{*}$ является $G$-инвариантным подпространством в алгебре $k[V]$.

ТеОРемА 7. Пусть $G$ - редуктивная алгебраическая группа, $V$ - конечномерный алгебраический $G$-модуль, для которого $k[V]^{G}=k, u \sigma \in \operatorname{Aut}_{G} V$. Тогда $\sigma \in \mathrm{GL}(V)$, если

(i) $V^{*}$ есть сумма некоторых изотипных компонент $G$-модуля $k[V]$.

Eсли группа $G$ полупроста, $L_{1}, \ldots, L_{s}$ - все попарно неизоморфные слагаемьце, встречающиеся в разложении $G$-модуля $V$ в сумму простых $G$-модулей, $m_{i}$ - кратность $G$-модуля $L_{i}$ в $V$ и $H$ - стабилизотор точки общего положения в $V$, то условие (i) әквивалентно условию

(ii) $m_{i}=\operatorname{dim} L_{i}^{H}$ для всех $i$.

ДокАЗАТЕЛЬСТво. Поскольку $\sigma^{*}$ является автоморфизмом $G$-модуля $k[V]$, каждая изотипная компонента $G$-модуля $k[V]$ инвариантна относительно $\sigma^{*}$. Значит, из (i) следует, что $\sigma^{*}\left(V^{*}\right)=V^{*}$, т.е. $\sigma \in \mathrm{GL}(V)$.

Пусть теперь группа $G$ полупроста. Тогда ввиду $k[V]^{G}=k$ многообразие $V$ содержит открытую плотную $G$-орбиту $\mathscr{O}$ и $V \backslash \mathscr{O}$ имеет в $V$ коразмерность, не меньшую 2 (см. [29, теорема 3.3 , следствие из теоремы 2.3 и теорема 3.1$])$. Следовательно, вложение $\iota: \mathscr{O} \hookrightarrow V$ индуцирует $G$-изоморфизм алгебр $\iota^{*}: k[V] \longrightarrow k[\mathscr{O}]$. Теперь второе утверждение теоремы 7 вытекает из двойственности $\Phi$ робениуса, согласно которой кратность $G$-модуля $L_{i}^{*}$ в $k[\mathscr{O}]$ равна $\operatorname{dim} L_{i}^{H}$.

СлЕДСТВИЕ 2. Пусть в обозначениях теоремы 7 группа $G$ полупроста, а модуль $V$ прост. Тогда следующие свойства эквивалентны:

(i) любой $G$-эквивариантный автоморфизм алгебраического многообразия $V$ является умножением на скаляр;

(ii) $\operatorname{Aut}_{G} V \subset \mathrm{GL}(V)$;

(iii) $\operatorname{dim} V^{H}=1$;

(iv) $\operatorname{dim} N_{G}(H) / H=1$.

ДокАЗАТЕЛЬСТво. Зафиксируем точку $v \in \mathscr{O}^{H}$. Так как орбита $\mathscr{O}$ открыта в $V$, то $\operatorname{dim} V^{H}=\operatorname{dim} \mathscr{O}^{H}$. Из $G_{v}=H$ следует, что $N_{G}(H) \cdot v=\mathscr{O}^{H}$ и многообразия $N_{G}(H) \cdot v$ и $N_{G}(H) / H$ эквивариантно изоморфны. Это дает (iii) $\Leftrightarrow(\mathrm{iv})$.

(i) $\Leftrightarrow$ (ii) вытекает из леммы Шура.

(iii) $\Rightarrow$ (ii) вытекает из теоремы 7 .

(i) $\Rightarrow$ (iv). Поскольку $\iota^{*}$ - изоморфизм, ограничение на орбиту $\mathscr{O}$ определяет изоморфизм между группами $\operatorname{Aut}_{G} V$ и $\operatorname{Aut}_{G} \mathscr{O}$. Следовательно, по теореме 6 орбита $N_{G}(H) \cdot v$ совпадает с одномерной орбитой точки $v$ относительно группы умножений на скаляры.

ЗАмечАние 1 . В общем случае, когда группа $G$ полупроста и $k[V]^{G}=k$, но $G$-модуль $V$ не обязательно прост, эти рассуждения и теорема 6 показывают, что:

(a) ограничение на орбиту $\mathscr{O}$ определяет изоморфизм между группой $\operatorname{Aut}_{G} V$ и группой $\operatorname{Aut}_{G} \mathscr{O}$, а потому и группой $N_{G}(H) / H$; 
(b) $\operatorname{dim} N_{G}(H) / H=\operatorname{dim} V^{H}$.

Утвеждения (a) и (b) аналогичным методом были доказаны в работе [20], появившейся одновременно с препринтом настояшей работы [28].

ЗАмечАниЕ 2. Для связных полупростых групп $G$ имеется явная классификация всех таких конечномерных алгебраических $G$-модулей $V$, что $k[V]^{G}=k$, и либо группа $G$ проста, либо модуль $V$ прост. В этих случаях найден $G$-стабилизатор $H$ точки общего положения в $V$ (см. [31], [13], [6], [7]). Подробнее: поскольку действие группы $G$ на пространстве $V$ не стабильно, группа $H$ бесконечна [25]. В [6], $[7]$ классифицированы все такие пары $(G, V)$, что $G$-стабилизотор $G_{*}$ точки общего положения в $V$ бесконечен и либо группа $G$ проста, либо модуль $V$ прост, и в этих случаях описана алгебра Ли $\operatorname{Lie}\left(G_{*}\right)$ (а в некоторых из них - также алгебра Ли $\operatorname{Lie}\left(N_{G}\left(G_{*}\right) / G_{*}\right)$ и подпространство $\left.V^{G_{*}}\right)$. Случаи, когда $k[V]^{G}=k$, выделяются из этой классификации условием $\operatorname{dim} V=\operatorname{dim} G-\operatorname{dim} \operatorname{Lie}\left(G_{*}\right)$. Вместе со следствием из теоремы 7 сказанное сводит вопрос о нахождении примеров простых $G$-модулей $V$, обладаюших нелинейными $G$-эквивариантными автоморфизмами, к задаче выгислительного характера. В статье [20] в классе простых модулей найден пример, в котором модуль имеет минимальную размерность.

2.6. Напомним, что конечномерный алгебраический модуль $V$ редуктивной алгебраической групшы $G$ называется сферическим, если пространство $V$ содержит открытую плотную орбиту борелевской подгрупшы группы $G$. Это эквивалентно тому, что $G$-модуль $k[V]$ не имеет кратностей, т.е. его ненулевые изотипные компоненты являются простыми $G$-модулями.

Теорема 8. Пусть $G$ - редуктивная алгебраическая группа и $V$ - сферический $G$-модуль. Тогда $\operatorname{Aut}_{G} V \subset \mathrm{GL}(V)$.

ДоКАЗАТЕЛЬСТВО. Поскольку каждая ненулевая изотипная компонента $G$-модуля $k[V]$ является простым $G$-модулем, выполнено условие (i) теоремы 7. Отсюда следует доказываемое утверждение.

Из леммы Шура вытекает

СлЕДСТвИЕ 3. Сохраним обозначения и предположсения теоремы 8. Если $G$-модуль $V$ прост, то группа $\operatorname{Aut}_{G} V$ состоит из умножений на скаляры.

2.7. Ниже через $A^{\times}$обозначается группа обратимых элементов кольца $A$.

Теорема 9. Пусть $G$ - алгебраическая группа, $V$ - конечномерный алгебраический $G$-модуль и $X$ - неприводимое алгебраическое многообразие. Рассмотрим действие группы $G$ на многообразии $Y:=V \times X$ через первый сомножитель. Пусть $H$ - алгебраическая подгруппа группь $\operatorname{Aut}_{G} Y$. Предположим, что:

(i) каждая G-орбита в пространстве $V$ содержит в своем замыкании нулевой вектор 0;

(ii) $\operatorname{Aut}_{G} V$ совпадает с әруппой умножений на скаляры;

(iii) $k[Y]^{\times} \subset k[Y]^{H}$. 
Тогда существуют такой алгебрачческий характер $\chi: H \longrightarrow k^{\times}$u такое действие группы $H$ на многообразии $X$, что естественное действие группы $G \times H$ на многообразии $Y$ задается формулой

$$
(g, h) \cdot(v, x)=(\chi(h) g \cdot v, h \cdot x), \quad(g, h) \in G \times H, \quad(v, u) \in Y .
$$

ДокАЗАТЕЛЬСТво. Из (i) следует, что $V^{G}=\{0\}$, откуда вытекает, что $Y^{G}=$ $\{0\} \times X$. Поэтому $\{0\} \times X$ является $H$-инвариантным подмногообразием в $Y$. Пусть

$$
\pi_{V}: Y \longrightarrow V, \quad \pi_{X}: Y \longrightarrow X
$$

- естественные проекции. Ограничение морфизма $\pi_{X}$ на подмногообразие $\{0\} \times X$ является изоморфизмом многообразий $\{0\} \times X$ и $X$. Значит, сушествует такое однозначно определенное действие группы $H$ на многообразии $X$, что этот изоморфизм является $H$-эквивариантным.

Рассмотрим точку $x \in X$. Из (i) следует, что точка $y \in Y$ лежит в слое $\pi_{X}^{-1}(x)$ тогда и только тогда, когда точка $(0, x)$ лежит в замыкании орбиты $G \cdot y$. Поскольку действие группы $H$ на многообразии $Y$ коммутирует с действием группы $G$, это показывает, что $\pi_{X}$ является $H$-эквивариантным морфизмом.

Отсюда вытекают два свойства. Во-первых, поскольку $\pi_{X}^{*}$ является $H$-эквивариантным вложением алгебры $k[X]$ в алгебру $k[Y]$, условие (iii) показывает, что

$$
k[X]^{\times} \subset k[X]^{H} .
$$

Во-вторых, для любого элемента $h \in H$ и любой точки $x \in X$ отображение $V \longrightarrow V, v \mapsto \pi_{V}(h \cdot(v, x))$, является $G$-эквивариантным автоморфизмом многообразия $V$. Следовательно, ввиду (ii) существует такой элемент $\mu_{h, x} \in k^{\times}$(зависяший от $h$ и $x$ ), что действие групшы $H$ на многообразии $Y$ задается формулой

$$
h \cdot(v, x)=\left(\mu_{h, x} v, h \cdot x\right), \quad h \in H, \quad v \in V, \quad x \in X .
$$

Из определения действия следует, что

$$
\mu_{h_{1} h_{2}, x}=\mu_{h_{1}, h_{2} \cdot x} \mu_{h_{2}, x}, \quad \mu_{e, x}=1, \quad h_{1}, h_{2} \in H, \quad x \in X .
$$

Для любого фиксированного элемента $h \in H$ функция $X \longrightarrow k^{\times}, x \mapsto \mu_{h, x}$, является элементом группы $k[X]^{\times}$. Поэтому из (1) вытекает, что

$$
\mu_{h_{1}, h_{2} \cdot x}=\mu_{h_{1}, x}, \quad h_{1}, h_{2} \in H, \quad x \in X .
$$

Из (3) и (4) теперь получаем, что

$$
\mu_{h_{1} h_{2}, x}=\mu_{h_{1}, x} \mu_{h_{2}, x}, \quad h_{1}, h_{2} \in H, \quad x \in X
$$


Таким образом, (5) показьвает, что для каждой фиксированной точки $x \in X$ отображение

$$
\chi_{x}: H \longrightarrow k^{\times}, \quad h \mapsto \mu_{h, x}
$$

является алгебраическим характером группы $H$. Поэтому ввиду $(2)$ дело сводится к тому, чтобы установить, что все эти характеры совпадают, т.е. $\chi_{x_{1}}=\chi_{x_{2}}$ для любых $x_{1}, x_{2} \in X$.

Чтобы доказать это, рассмотрим морфизм

$$
\tau: H \times X \longrightarrow k^{\times}, \quad \tau((h, x))=\mu_{h, x}, \quad h \in H, \quad x \in X .
$$

Пусть $H^{0}$ - связная компонента единицы группы $H$. Поскольку многообразие $H^{0} \times X$ неприводимо, а ограничение морфизма $\tau$ на многообразие $H^{0} \times X$ является элементом групшы $k\left[H^{0} \times X\right]^{\times}$, из [30] следует, что существуют такой алгебраический характер $\varepsilon: H^{0} \longrightarrow k^{\times}$и такая функция $\vartheta \in k[X]^{\times}$, что

$$
\tau((h, x))=\varepsilon(h) \vartheta(x), \quad h \in H^{0}, \quad x \in X .
$$

Подставляя $h=e$ в (8) и учитывая $(7),(3)$ и соотношение $\varepsilon(e)=1$, мы получаем, что $\vartheta \equiv 1$. Это означает, что ограничения всех характеров $\chi_{x}$ на группу $H^{0}$ равны $\varepsilon$ и, значит, равны друг другу. Поэтому если $x_{0} \in X$ - фиксированная точка, то для любой точки $x \in X$ образ характера $\chi_{x} \chi_{x_{0}}^{-1}: H \longrightarrow k^{\times}$лежит в группе

$$
F:=\left\{a \in k^{\times} \mid a^{\operatorname{ord}\left(H / H^{0}\right)}=1\right\} .
$$

Значит, образ отображения

$$
\tau^{\prime}: H \times X \longrightarrow k^{\times}, \quad \tau^{\prime}((h, x))=\tau((h, x)) \chi_{x_{0}}(h)^{-1}, \quad h \in H, \quad x \in X,
$$

тоже лежит в группе $F$. Поскольку многообразие $X$ неприводимо, а группа $F$ конечна, отсюда следует, что

$$
\tau^{\prime}\left(\left(h, x_{1}\right)\right)=\tau^{\prime}\left(\left(h, x_{2}\right)\right) \text { для любых } h \in H, \quad x_{1}, x_{2} \in X .
$$

Теперь ввиду (9), (7) и (6) мы получам, что $\tau^{\prime}\left(\left(h, x_{0}\right)\right)=1$ для любого $h \in H$. Вместе с (10) это показывает, что $\tau^{\prime} \equiv 1$. Следовательно, ввиду (9), (7) и (6) мы имеем $\chi_{x}=\chi_{x_{0}}$ для любой точки $x \in X$, как и утверждалось.

ЗАмЕчАнИЕ 3. Поскольку для любого неприводимого алгебраического многообразия $Z$ группа $k[Z]^{\times} / k^{\times}$является свободной абелевой конечного ранга, условие (iii) теоремы 9 автоматически выполнено, если группа $H$ связна и не имеет нетривиальных характеров. 
СлЕДСТВИЕ 4. Сохраним обозначения и условия теоремы 9. Пусть $X=$ $\mathbb{A}^{n}$. Если действие группь $H$ на многообразии $X$ линеаризуемо, то и естественное действие группь $G \times H$ на многообразии $Y$ линеаризуемо.

СлЕДСТвИЕ 5. Сохраним обозначения и условия теоремы 9. Пусть $X=$ $\mathbb{A}^{n}$. Предположим, что группа $H$ редуктивна. Тогда естественное действие группь $G \times H$ на многообразии $Y$ линеаризуемо в каждом из следующих случаев:

(i) $n \leqslant 2$;

(ii) $n=3$ и группа $H$ связна;

(iii) $n=4$ и әруппа $H$ связна и не является одно- или двумерным тором.

ДокАЗАТЕЛЬСтво. В случае (i) утверждение вытекает из следствия 4 и из п. 1.2, а в случаях (ii) и (iii) - соответственно из доказанных ниже теорем 1 и 2 (в их доказательствах используется только случай (i)).

2.8. В отношении автоморфизмов простых модулей имеет место

Теорема 10. Пусть $G$ - редуктивная алгебраическая группа и $\mathrm{V}$ - простой алгебраический $G$-модуль. Пусть $H$ - бесконечная редуктивная алгебраическая подгруппа в группе Aut $_{G} V$. Тогда естественное действие группы $G \times H$ на многообразии $V$ линеаризуемо и группа $H$ является одномерным тором.

ДокаЗАтельСтво. Можно считать, что $\operatorname{dim} V>1$. Тогда $V^{G}=\{0\}$ и, значит, $0 \in V^{H}$. Слайс-модулем $G$-многообразия $V$ в неподвижной точке 0 является сам $G$-модуль $V$, а если мы рассмотрим $V$ как $H$-многообразие, то этим слайс-модулем является векторное пространство $V$, снабженное некоторым линейным действием группы $H$, коммутируюшим с действием групшы $G$. Поскольку $V$ является простым $G$-модулем, из леммы Шура следует, что группа $H$ действует на этом последнем слайс-модуле умножениями на скаляры. Поскольку группа $H$ бесконечна и действует на многообразии $V$ эффективно, отсюда и из теоремы Луны об этальном слайсе [21] вытекает, что группа $H$ является одномерным тором и категорный фактор для рассматриваемого в теореме действия группы $H$ на многообразии $V$ является точкой. Следовательно, категорный фактор для естественного действия группы $G \times H$ на многообразии $V$ также является точкой. Доказываемое утверждение вытекает теперь из теоремы 4.

2.9. Если редуктивная алгебраическая группа $G$ действует на аффинном алгебраическом многообразии $X$ и $k[X]^{G}=k$, то $\operatorname{Aut}_{G}(X)$ - конечномерная алгебраическая группа, поскольку алгебра $k[X]$ порождена суммой конечного числа своих изотипных компонент и эта сумма ввиду $k[X]^{G}=k$ конечномерна. В качестве приложения получаем следуюший критерий линеаризуемости действия группы $G \times \operatorname{Aut}_{G}(V)$.

Теорема 11. Пусть $G$ - редуктивная алгебраическая группа $u V-$ такой простой алгебрачческий $G$-модуль, что $k[V]^{G}=k$. Тогда следующие свойства эквивалентны: 
(i) действие группы $G \times \operatorname{Aut}_{G}(V)$ на пространстве $V$ линеаризуемо;

(ii) группа $\operatorname{Aut}_{G}(V)$ является одномерным тором.

Если группа $G$ полупроста и действует на пространстве $V$ әффективно, а $H$ является $G$-стабилизатором точки общего положения в пространстве $V$, то эти свойства эквивалентны любому из следующих:

(iii) действие группы $G \times \operatorname{Aut}_{G}(V)$ на пространстве $V$ линейно;

(iv) $\operatorname{dim} V^{H}=1$;

(v) $\operatorname{dim} N_{G}(H) / H=1$;

(vi) группа $N_{G}(H) / H$ является одномерным тором.

ДокАЗАТЕЛЬСтво. (i) $\Rightarrow$ (ii). Поскольку в группе $\mathrm{GL}(V)$ централизатор любой редуктивной подгрупшы редуктивен (см., например, [23, предложение 3.6$]$ ), из (i) следует, что $\operatorname{Aut}_{G}(V)$ - редуктивная группа. Она бесконечна, так как содержит все умножения на скаляры. Теперь (ii) следует из теоремы 10.

(ii) $\Rightarrow$ (i) вытекает из теоремы 10 .

(ii) $\Leftrightarrow$ (vi) следует из замечания $1,(\mathrm{a})$.

(iii) $\Leftrightarrow(\mathrm{iv}) \Leftrightarrow(\mathrm{v})$ вытекает из следствия 2 .

Импликации (vi) $\Rightarrow(\mathrm{v})$ и (iii) $\Rightarrow$ (i) очевидны.

2.10. Теорема 11 (см. также замечание 2) доставляет способ построения примеров нелинеаризуемых действий бесконечных нередуктивных неразрешимых алгебраических групп с непустым множеством неподвижных точек. А именно, если $V$ - такой простой модуль связной полупростой алгебраической группы $G$, что $k[V]^{G}=k$ и $\operatorname{dim} N_{G}(H) / H>1$, где $H$ - стабилизатор точки общего положения в $V$, то действие группы $G \times N_{G}(H) / H$ на $V$ нелинеаризуемо (по теореме 10 в этом случае группа $G \times N_{G}(H) / H$ нередуктивна). Например, рассмотренное в $[20, \S 3]$ действие групшы $G=\mathrm{SL}_{3} \times \mathrm{SL}_{5} \times \mathrm{SL}_{13} \times\left(k^{*} \ltimes k_{+}\right)$на $\mathbb{A}^{195}$ является таким примеpoм.

\section{§ 3. Доказательство теоремы 1}

3.1. Пусть $G$ - связная редуктивная алгебраическая группа, действуюшая на пространстве $\mathbb{A}^{3}$.

Еслигруппа $G$ полупроста, утверждение теоремы 1 доказано в [19]. Еслигруппа $G$ - тор, то можно считать, что $\operatorname{dim} G=1,2,3$, и в этих случаях утверждение доказано соответственно в [12], [4] и [3].

3.2. Таким образом, дело сводится к тому, чтобы доказать, что если $Z$ - неединичный алгебраический тор, $S$ - односвязная полупростая алгебраическая группа и группа $G:=Z \times S$ действует на пространстве $\mathbb{A}^{3}$ с конечным ядром неэффективности, то действие линеаризуемо.

3.3. Всякое действие связной полупростой алгебраической группы ранга $\geqslant 2$ на пространстве $\mathbb{A}^{3}$ имеет открытую плотную орбиту (см. [26], а также [19, замечание 5.2]). Поэтому если $S \neq \mathrm{SL}_{2}(k)$, то группа $G$ имеет открытую плотную 
орбиту в пространстве $\mathbb{A}^{3}$. В этом случае действие групшы $G$ на пространстве $\mathbb{A}^{3}$ линеаризуемо по теореме 4 .

3.4. Таким образом, можно считать, что $S=\mathrm{SL}_{2}(k)$.

Поскольку любое действие групшы $S$ на пространстве $\mathbb{A}^{3}$ линеаризуемо, $S$-многообразие $\mathbb{A}^{3}$ совпадает с точностью до изоморфизма либо с $V_{2}$, либо с $V_{1} \oplus V_{0}$ (см. п. 1.9). Если $\mathbb{A}^{3}$ - это $V_{2}$, то утверждение теоремы 1 вытекает из теоремы 10 . Если же $\mathbb{A}^{3}$ - это $V_{1} \oplus V_{0}$, то оно вытекает из теоремы 8 и следствия 5 (случай (i)), поскольку $V_{1}$ является сферическим $\mathrm{SL}_{2}(k)$-модулем.

\section{§ 4. Доказательство теоремы 2: первый шаг}

4.1. Пусть $G$ - связная редуктивная алгебраическая группа, действуюшая на пространстве $\mathbb{A}^{4}$ и отличная от одно- или двумерного тора.

Если группа $G$ полупроста, то утверждение теоремы 2 доказано в [24]. Если группа $G$ - тор, то можно считать, что $\operatorname{dim} G \leqslant 4$ и, значит, $\operatorname{dim} G=3,4$. В этих случаях утверждение теоремы 2 доказано соответственно в [4] и [3].

4.2. Таким образом, все сводится к тому, чтобы показать, что если $Z$ - неединичный алгебраический тор, $S$ - односвязная полупростая алгебраическая группа и группа $G:=Z \times S$ действует на пространстве $\mathbb{A}^{4}$ с конечным ядром неэффективности, то действие линеаризуемо. Мы можем (и будем) считать, что тор $Z$ действует на пространстве $\mathbb{A}^{4}$ эффективно.

4.3. Поскольку ограничение действия на группу $S$ линеаризуемо, мы можем зафиксировать подходящую линейную структуру на пространстве $\mathbb{A}^{4}$ и считать $\mathbb{A}^{4}$ некоторым $S$-модулем $V$. Поскольку группа $S$ действует на $V$ с конечным ядром неэффективности, из [26] следует, что с точностью до перехода к двойственному модулю пара $(S, V)$ содержится в следуюшем списке (ниже мы сохраняем обозначения $\S 3$ и обозначаем через $R(\lambda)$ и $\omega_{i}$ соответственно простой $S$-модуль со старшим весом $\lambda$ и $i$-й фундаментальньй вес в нумерации Бурбаки):

$$
\begin{array}{cl}
\left(\mathrm{SL}_{2}(k), V_{3}\right), \quad\left(\mathrm{SL}_{2}(k), V_{2} \oplus V_{0}\right), & \left(\mathrm{SL}_{2}(k), V_{1} \oplus V_{1}\right), \quad\left(\mathrm{SL}_{2}(k), V_{1} \oplus V_{0} \oplus V_{0}\right), \\
\left(\mathrm{SL}_{3}(k), R\left(\omega_{1}\right) \oplus R(0)\right), \quad\left(\mathrm{SL}_{4}(k), R\left(\omega_{1}\right)\right), \quad\left(\mathrm{Sp}_{4}(k), R\left(\omega_{1}\right)\right) \\
\left(\mathrm{SL}_{2}(k) \times \mathrm{SL}_{2}(k), V_{1} \otimes V_{1}\right), \quad\left(\mathrm{SL}_{2}(k) \times \mathrm{SL}_{2}(k),\left(V_{1} \otimes V_{0}\right) \oplus\left(V_{0} \otimes V_{1}\right)\right) .
\end{array}
$$

Мы рассмотрим отдельно все эти возможности.

4.4. Если $(S, V)$ - одна из пар $\left(\mathrm{SL}_{2}(k) \times \mathrm{SL}_{2}(k),\left(V_{1} \otimes V_{0}\right) \oplus\left(V_{0} \otimes V_{1}\right)\right),\left(\mathrm{SL}_{4}(k)\right.$, $\left.R\left(\omega_{1}\right)\right)$ и $\left(\mathrm{Sp}_{4}(k), R\left(\omega_{1}\right)\right)$, то в $V$ имеется открытая плотная $S$-орбита, и потому доказываемое утверждение вытекает из теоремы 4.

4.5. Если $(S, V)=\left(\mathrm{SL}_{2}(k), V_{1} \oplus V_{0} \oplus V_{0}\right)$ или $(S, V)=\left(\mathrm{SL}_{3}(k), R\left(\omega_{1}\right) \oplus\right.$ $R(0))$, то, поскольку $V_{1}$ является сферическим $\mathrm{SL}_{2}(k)$-модулем, а $R\left(\omega_{1}\right)$ - сферическим $\mathrm{SL}_{3}(k)$-модулем, доказываемое утверждение вытекает из теоремы 8 и следствия 5,(i). 
4.6. Если $(S, V)=\left(\mathrm{SL}_{2}(k), V_{3}\right)$ или $(S, V)=\left(\mathrm{SL}_{2}(k) \times \mathrm{SL}_{2}(k), V_{1} \otimes V_{1}\right)$, доказываемое утверждение вытекает из теоремы 10.

4.7. Оставшиеся случаи $(S, V)=\left(\mathrm{SL}_{2}(k), V_{2} \oplus V_{0}\right),\left(\mathrm{SL}_{2}(k), V_{1} \oplus V_{1}\right)$ рассмотрены в двух следуюших параграфах.

\section{§ 5. Доказательство теоремы 2: второй шаг}

5.1. Рассмотрим первый из оставшихся случаев:

$$
(S, V)=\left(\mathrm{SL}_{2}(k), V_{2} \oplus V_{0}\right)
$$

5.2. Поскольку $V_{2} / / S=\mathbb{A}^{1}$, мы имеем

$$
V / / S=\left(V_{2} / / S\right) \times V_{0}=\mathbb{A}^{2} .
$$

Ввиду (11) многообразие $V / / G=(V / / S) / / Z$ максимум двумерно.

5.3. Если оно нульмерно (и потому является точкой), утверждение теоремы 2 следует из теоремы 4.

5.4. Если оно двумерно, обший слой морфизма $\pi_{V / / S, Z}$ нульмерен. Поэтому ввиду связности тор $Z$ тривиально действует на этом слое. Значит, он тривиально действует на многообразии $V / / S$. В частности, тор $Z$ тривиально действует на прямой $\pi_{V / / S, Z}\left(\{0\} \times V_{0}\right)$. Эта прямая является изоморфным образом прямой $\{0\} \times$ $V_{0}$ относительно $Z$-эквивариантного морфизма $\pi_{V / / S, Z}$. Поскольку $\{0\} \times V_{0}=$ $V^{G}$, прямая $\{0\} \times V_{0}$ инвариантна относительно $Z$. Поэтому каждая ее точка неподвижна относительно $Z$. Значит, в этом случае $\{0\} \times V_{0}=V^{G}$.

Пусть $T_{x}$ - слайс-модуль $G$-многообразия $V$ в точке $x \in\{0\} \times V_{0}$. Тогда, как $S$-модуль, $T_{x}=V_{2} \oplus V_{0}$. Поскольку $S$-подмодули $T_{x, 2}=V_{2} \times\{0\}$ и $T_{x, 0}=$ $\{0\} \times V_{0}$ в $T_{x}$ просты и неизоморфны, они $Z$-инвариантны, и по лемме Шура тор $Z$ действует на каждом из них умножениями на скаляры. Поскольку $T_{x, 0}$ является касательным пространством к многообразию $V^{G}$ в точке $x$, действие тора $Z$ на $T_{x, 0}$ тривиально. С другой стороны, действие тора $Z$ на $T_{x, 2}$ нетривиально: иначе бы действие тора $Z$ на $T_{x}$ было тривиальным, а тогда по теореме Луны об этальном слайсе [21] было бы тривиальным и действие тора $Z$ на $V$, что не так (см. п. 4.2).

Из сказанного следует, что действие группы $G$ на $T_{x}$ - это fixed pointed-действие. Из теоремы Луны об этальном слайсе [21] вытекает, что тогда и действие группы $G$ на $V$ тоже fixed pointed. Поэтому в рассматриваемом случае утверждение теоремы 2 вытекает из теоремы 5 .

5.5. Таким образом, следует рассмотреть оставшийся случай, когда многообразие $V / / G$ одномерно. Поскольку морфизм $\pi_{V, G}$ сюръективен, а всякая неприводимая унирациональная нормальная аффинная кривая, на которой нет непостоянных регулярных функций, есть $\mathbb{A}^{1}$, мы имеем

$$
(V / / S) / / Z=\mathbb{A}^{1} .
$$


Чтобы завершить доказательство теоремы 2 в этом случае, мы найдем в алгебpe $k[V]$ четырехмерное $G$-инвариантное линейное подпространство, порождающее $k[V]$ как $k$-алгебру. Поскольку $\operatorname{dim} V=4$, его существование эквивалентно линеаризуемости действия групшы $G$ на пространстве $V$.

5.6. ПРЕДЛОЖЕНИЕ 1. В алгебре $k[V]^{S}$ существуют такие два непостоянных $Z$-полуинварианта $f_{1}$ и $f_{2}$, что:

(i) $k[V]^{S}=k\left[f_{1}, f_{2}\right]$;

(ii) $k[V]^{G}=k[f]$, где $f=f_{1}^{a_{1}} f_{2}^{a_{2}}$ для некоторьх иисел $a_{1}, a_{2} \in \mathbb{N}$;

(iii) каждая ненулевая изотипная компонента $Z$-модуля $k[V]^{S}$ имеет вид $f_{1}^{b_{1}} f_{2}^{b_{2}} k[V]^{G}$ для некоторых чисел $b_{1}, b_{2} \in \mathbb{N}$.

ДокаЗАТЕЛЬСтво. Сушествование $Z$-полуинвариантов $f_{1}$ и $f_{2}$, для которых выполнено (i), вытекает из (11) и линеаризуемости действий алгебраического тора на плоскости $\mathbb{A}^{2}$.

Пусть $\chi_{i} \in \operatorname{Hom}\left(Z, k^{\times}\right)$- вес $Z$-полуинварианта $f_{i}$. Рассмотрим в группе $\mathbb{Z}^{2}$ подгруппу

$$
\mathrm{D}_{0}:=\left\{\left(d_{1}, d_{2}\right) \in \mathbb{Z}^{2} \mid \chi_{1}^{d_{1}} \chi_{2}^{d_{2}}=\mathrm{id}\right\} .
$$

Линейной оболочкой множества $\left\{f_{1}^{d_{1}} f_{2}^{d_{2}} \mid\left(d_{1}, d_{2}\right) \in \mathrm{D}_{0} \cap \mathbb{N}^{2}\right\}$ является алгебра $\left(k[V]^{S}\right)^{Z}=k[V]^{G}$. Поскольку группа $Z$ связна, $\mathrm{D}_{0}$ является насьщенной подгруппой в $\mathbb{Z}^{2}$. Ввиду (12) $\mathrm{rk}_{0}=1$ и

$$
\mathrm{D}_{0} \cap \mathbb{N}^{2} \neq\{(0,0)\}
$$

Следовательно, подгруппа $\mathrm{D}_{0}$ порождена некоторым элементом $\left(a_{1}, a_{2}\right) \in \mathbb{N}^{2}$. Это доказывает (ii).

Пусть $I$ - ненулевая изотипная компонента $Z$-модуля $k[V]^{S}$, весом которой является $\chi \in \operatorname{Hom}\left(Z, k^{\times}\right)$. Ввиду (i) компонента $I$ является линейной оболочкой множества $\left\{f_{1}^{d_{1}} f_{2}^{d_{2}} \mid\left(d_{1}, d_{2}\right) \in \mathrm{D}_{\chi} \cap \mathbb{N}^{2}\right\}$, где

$$
\mathrm{D}_{\chi}:=\left\{\left(d_{1}, d_{2}\right) \in \mathbb{Z}^{2} \mid \chi_{1}^{d_{1}} \chi_{2}^{d_{2}}=\chi\right\}
$$

Поскольку $\mathrm{D}_{\chi}$ является смежньм классом группы $\mathbb{Z}^{2}$ по подгруппе $\mathrm{D}_{0}$, из (13) и (ii) следует, что $I$ имеет указанный в (iii) вид, где $\left(b_{1}, b_{2}\right)$ - элемент из $\mathrm{D}_{\chi} \cap$ $\left(\mathbb{N}^{2} \backslash\{(0,0)\}\right)$ с минимальной суммой координат.

5.7. Рассмотрим в пространстве $V^{*}$ линейные подпространства

$$
L:=\pi_{2}^{*}\left(V_{2}^{*}\right), \quad M:=\pi_{0}^{*}\left(V_{0}^{*}\right),
$$

где $\pi_{2}: V=V_{2} \oplus V_{0} \rightarrow V_{2}, \pi_{0}: V=V_{2} \oplus V_{0} \rightarrow V_{0}$ - естественные проекции. Подпространства $L$ и $M$ являются $S$-подмодулями $S$-модуля $V^{*}$ и

$$
\begin{gathered}
V^{*}=L \oplus M, \\
M \subset k[V]^{S} .
\end{gathered}
$$


ПРЕДЛОЖЕНИЕ 2. Подпространство $L$ является $G$-инвариантным, $и$ тор $Z$ действует на нем умножениями на скаляры.

ДокАЗАТЕльство. Зафиксируем в $S$ борелевскую подгруппу $B$. Пусть $U$ - ее унипотентный радикал и $\varepsilon$ - старший вес $S$-модуля $V_{1}$ относительно $B$. Тогда $\varepsilon^{d}$ старший вес модуля $V_{d}$ относительно $B$. Для любого алгебраического $S$-модуля $M$ и любого числа $d \in \mathbb{N}$ обозначим через $M_{d}$ изотипную компоненту типа $V_{d}$ модуля $M$.

Пусть $d \in \mathbb{N}$. Тогда $k\left[V_{2}\right]_{d}^{U}$ является конечно порожденным $k\left[V_{2}\right]^{S}$-модулем без кручения. Поскольку $V_{2} / / S=\mathbb{A}^{1}$, этот модуль является свободным конечного ранга $m_{d}$, а морфизм $\pi_{V_{2}, S}-$ плоским. Из последнего свойства следует, что для каждого схемного слоя $\pi_{V_{2}, S}^{-1}(x), x \in V_{2} / / S$, имеет место равенство

$$
m_{d}=\frac{\operatorname{dim} k\left[\pi_{V_{2}, S}^{-1}(x)\right]_{d}}{\operatorname{dim} V_{d}}
$$

(см. [29]). Пусть $\pi_{V_{2}, S}^{-1}(x)$ в $(17)$ является слоем общего положения. Этот слой приведен и изоморфен многообразию $S / T$, где $T$ - максимальный тор группы $S$ (в действительности в рассматриваемом случае каждый слой приведен; см. первый абзац п. 5.9). Значит, по двойственности Фробениуса правая часть в (17) равна $\operatorname{dim} V_{d}^{T}$ (см. [29]). Это дает

$$
m_{d}= \begin{cases}1, & \text { если } d \text { четно, } \\ 0, & \text { если } d \text { нечетно }\end{cases}
$$

Ввиду (14) и (15) подалгебра алгебры $k[V]$, порожденная подпространством $L$, инвариантна относительно $S$ и $S$-изоморфна алгебре $k\left[V_{2}\right]$. Ввиду (16) это показывает, что пространство $k[V]_{d}^{U}$ является свободным $k[V]^{S}$-модулем ранга $m_{d}$, заданного формулой (18). Таким образом, для любого четного натурального числа $d$ существует такой ненулевой элемент $h_{d}$, что

$$
k[V]_{d}^{U}=h_{d} k[V]^{S}
$$

$\mathrm{C}$ точностью до скалярного множителя элемент $h_{d}$ определен однозначно как элемент из алгебры $k[V]_{d}^{U}$, не имеющий непостоянных $S$-инвариантных делителей. В самом деле, $k[V]_{d}^{U}$ - это подпространство всех $B$-полуинвариантов веса $\varepsilon^{d}$ в алгебре $k[V]$. Поэтому из равенства $h_{d}=p q, p \in k[V]^{S}$, следует, что $q \in k[V]_{d}^{U}$. В силу (19) это дает $q=h_{d} r$, где $r \in k[V]^{S}$. Значит, $r p=1$, и потому $p$ является константой. Утверждение теперь вытекает из (19).

Поскольку тор $Z$ действует $S$-эквивариантными автоморфизмами алгебры $k[V]$, эта характеризация элементов $h_{d}$ показывает, что каждый элемент $h_{d}$ является $Z$-весовым вектором.

Далее, пусть $d=2$. Из (15) следует, что каждый ненулевой элемент пространства $L$ является простым элементом факториального кольца $k[V]$. В частности, он не имеет непостоянных $S$-инвариантных делителей. Поскольку $S$-модули $L$ и 
$V_{2}$ изоморфны и $L^{U} \neq 0$, данная вьше характеризация элементов $h_{d}$ показывает, что $h_{2} \in L$. Для завершения доказательства остается заметить, что элемент $h_{2}$ является $Z$-весовым вектором, а линейной оболочкой его $S$-орбиты является пространство $L$.

5.8. Зафиксируем в пространстве $L$ базис $t_{1}, t_{2}, t_{3}$. Тогда из (15) и (16) следует, что $k[V]=k[V]^{S}\left[t_{1}, t_{2}, t_{3}\right]$ и, значит, по предложению 1

$$
k[V]=k\left[t_{1}, t_{2}, t_{3}, f_{1}, f_{2}\right] .
$$

Покажем, что в правой части равенства $(20)$ можно убрать либо $f_{1}$, либо $f_{2}$, сохраняя это равенство. Поскольку линейная оболочка множества $t_{1}, t_{2}, t_{3}, f_{i}$ четырехмерна и по предложениям 1 и $2 G$-инварианта, это завершит доказательтство теоремы 2 в рассматриваемом случае (см. п. 5.5).

5.9. Пусть $h$ - однородная образующая (степени 2) алгебры $k\left[V_{2}\right]^{S}$. Поскольку группа $S$ связна и полупроста, простые множители любого элемента из $k\left[V_{2}\right]^{S}$ в факториальном кольце $k\left[V_{2}\right]$ содержатся в $k\left[V_{2}\right]^{S}$. Отсюда следует, что для любой константы $\alpha \in k$ полином $h+\alpha$ является простым элементом кольца $k\left[V_{2}\right]$.

Ввиду (14), (15) можно отождествить алгебру $k\left[V_{2}\right]$ с подалгеброй алгебры $k[V]$, порожденной подпространством $L$, и при этом отождествлении для любой константы $\alpha \in k$ полином $h+\alpha$ также является простым элементом факториального кольца $k[V]$.

Из определения элементов $t_{1}, t_{2}, t_{3}$ вытекает, что

$$
h \in k\left[t_{1}, t_{2}, t_{3}\right] .
$$

Поскольку элемент $h$ однороден, предложение 2 показывает, что элемент $h$ содержится в некоторой изотипной компоненте $Z$-модуля $k[V]^{S}$. Значит, по предложению 1

$$
h=f_{1}^{b_{1}} f_{2}^{b_{2}}\left(\alpha_{n}\left(f_{1}^{a_{1}} f_{2}^{a_{2}}\right)^{n}+\alpha_{n-1}\left(f_{1}^{a_{1}} f_{2}^{a_{2}}\right)^{n-1}+\cdots+\alpha_{0}\right)
$$

для некоторых $n \in \mathbb{N},\left(b_{1}, b_{2}\right),\left(a_{1}, a_{2}\right) \in \mathbb{N}^{2}, \alpha_{i} \in k, \alpha_{n} \neq 0$.

Поскольку элемент $h+\alpha$ прост для любой константы $\alpha \in k$, а $f_{1}, f_{2}$-непостоянные элементы алгебры $k[V]$, из (22) следует, что имеет место один из следующих случаев:

(i) $b_{1}=1, b_{2}=0, n=0$;

(ii) $b_{1}=0, b_{2}=1, n=0$;

(iii) $b_{1}=0, b_{2}=0, n=1, a_{1}=1, a_{2}=0$;

(iv) $b_{1}=0, b_{2}=0, n=1, a_{1}=0, a_{2}=1$.

Ввиду (21) и (22) в случаях (i) и (iii) мы имеем включение $f_{1} \in k\left[t_{1}, t_{2}, t_{3}\right]$, а в случаях (ii) и (iv) - включение $f_{2} \in k\left[t_{1}, t_{2}, t_{3}\right]$. Это завершает доказательство теоремы 2 в случае $(S, V)=\left(\mathrm{SL}_{2}(k), V_{2} \oplus V_{0}\right)$. 


\section{§ 6. Доказательство теоремы 3: последний шаг}

6.1. Расмотрим последний оставшийся случай

$$
(S, V)=\left(\mathrm{SL}_{2}(k), V_{1} \oplus V_{1}\right)
$$

6.2. Из (23) следует, что $V / / S=\mathbb{A}^{1}$. Значит, есть лишь две возможности: либо в многообразии $V / / S$ имеется открытая плотная $Z$-орбита, либо тор $Z$ действует на многообразии $V / / S$ тривиально.

Если имеет место первая возможность, то фактор $V / / G=(V / / S) / / Z$ является точкой, и утверждение теоремы 2 следует из теоремы 4 . Остается рассмотреть вторую возможность.

6.3. Итак, предположим, что тор $Z$ действует тривиально на факторе $V / / S$. Тогда каждый слой морфизма $\pi_{V, S}$ инвариантен относительно $Z$ и

$$
V / / G=(V / / S) / / Z=(V / / Z) / / S=\mathbb{A}^{1} .
$$

6.4. $S$-стабилизатор точки общего положения в пространстве $V$ тривиален и тор $S$ действует транзитивно на слое общего положения $F$ морфизма $\pi_{V, S}$. Поэтому существует изоморфизм $S$-многообразий

$$
F \simeq \mathrm{SL}_{2}(k)
$$

(группа $S=\mathrm{SL}_{2}(k)$ действует на многообразии из правой части формулы $(25)$ с помошью левых сдвигов).

Поскольку группа $S$ транзитивно действует на многообразии $F$ и это действие коммутирует с действием групшы $Z$, все $Z$-орбиты в $F$ имеют одинаковую размерность, равную, скажем, $d$. В частности, все они замкнуты в $V$. Ввиду п. 4.2 мы имеем $d \geqslant 1$.

Таким образом, $Z$-орбита общего положения в пространстве $V$ замкнута и имеет размерность $d$. Поэтому

$$
\operatorname{dim} V / / Z=4-d
$$

6.5. Пусть $D$ - слой общего положения морфизма $\pi_{V / / Z, S}$. Из (24) и (26) следует, что

$$
\operatorname{dim} D=3-d .
$$

Согласно общим свойствам категорных факторов многообразие $D$ содержит единственную замкнутую $S$-орбиту. Она аффинна и ввиду (27) имеет размерность, не превосходящую 2. Поскольку не существует одномерных аффинных однородных пространств группы $\mathrm{SL}_{2}$, это показывает, что $D$ является либо точкой, либо поверхностью. 
6.6. Если имеет место первый случай, то $d=3$. Поскольку $\operatorname{dim} F=3$, это означает, что группа $Z$ действует на слое $F$ транзитивно. Из того, что $Z$ - тор, следует, что алгебраическое многообразие $F$ изоморфно $\mathbb{A}_{*}^{3}:=\mathbb{A}_{*} \times \mathbb{A}_{*} \times \mathbb{A}_{*}$, где $\mathbb{A}_{*}=\mathbb{A}^{1} \backslash\{0\}$. Но это противоречит $(25)$, поскольку $k\left[\mathbb{A}_{*}^{3}\right]^{\times} \neq k^{\times}$, а $k\left[\mathrm{SL}_{2}(k)\right]^{\times}=$ $k^{\times}$(см. [30]). Значит, этот случай невозможен.

\section{7. Итак,}

$$
d=1 .
$$

Поскольку для любого действия редуктивной алгебраической группы на гладком неприводимом аффинном алгебраическом многообразии стабилизаторы точек общего положения сопряжены, из эффективности действия тора $Z$ на пространстве $V$ (см п. 4.2) следует, что $Z$-стабилизатор точки общего положения в пространстве $V$ тривиален. Отсюда и из (28) вытекает, что

$$
\operatorname{dim} Z=1 \text {. }
$$

6.8. Мы можем (и будем) отождествлять $S$-многообразие $V$ с пространством Mat $_{2 \times 2}(k)$ всех матриц размера $2 \times 2$ с коэффициентами из поля $k$, на котором группа $S=\mathrm{SL}_{2}(k)$ действует левыми умножениями.

Пусть $x_{p q}, 1 \leqslant p, q \leqslant 2,-$ стандартная $p q$-я координатная функция на пространстве $V$, т.е.

$$
x_{p q}(v)=a_{p q}, \quad v=\left[a_{i j}\right]:=\left[\begin{array}{ll}
a_{11} & a_{12} \\
a_{21} & a_{22}
\end{array}\right] .
$$

Тогда

$$
k[V]^{S}=k[\operatorname{det}], \quad \operatorname{det}=x_{11} x_{22}-x_{12} x_{21} .
$$

Слоями морфизма $\pi_{V, S}$ являются гиперповерхности $F_{c}:=\{v \in V \mid \operatorname{det}(v)=$ $c\}, c \in k$. Они $G$-инвариантны. Если $c \neq 0$, то действие группы $S$ на слое $F_{c}$ транзитивно, а $S$-стабилизатор точки из этого слоя тривиален.

Рассмотрим также действие группы $S=\mathrm{SL}_{2}(k)$ напространстве $V=\operatorname{Mat}_{2 \times 2}(k)$ с помощью правых умножений. Оно коммутирует с исходным действием. Поскольку $\operatorname{det}(a b)=\operatorname{det}(a) \operatorname{det}(b)$ для любых матриц $a, b \in \mathrm{Mat}_{2 \times 2}(k)$, каждый слой $F_{c}$ инвариантен также и относительно этого действия группы $S$.

6.9. Отсюда и из теоремы 6 вытекает, что для любого элемента $c \in k, c \neq 0$, сушествует такой гомоморфизм

$$
\lambda_{c}: Z \longrightarrow \mathrm{SL}_{2}(k)
$$

что действие группы $Z$ на слое $F_{c}$ пропускается через $\lambda_{c}$, т.е.

$$
z \cdot v=v \lambda_{c}(z), \quad z \in Z, \quad v \in F_{c}
$$

(в правой части (32) рассматривается обычное матричное умножение). 
ПРЕДЛОЖЕНИЕ 3. Гомоморфизмы $\lambda_{c}$ и их зависимость от параметра $c$ являются алгебраическими, т.е. отображсение

$$
\lambda: Z \times \mathbb{A}_{*} \longrightarrow \mathrm{SL}_{2}(k), \quad(z, c) \mapsto \lambda_{c}(z),
$$

является морфизмом.

ДоКАЗАТЕЛЬСТво. Открытое множество $\Omega:=V \backslash F_{0}$ инвариантно относительно группы $G$. Ввиду (32) отображение

$$
\alpha: Z \times \Omega \longrightarrow \Omega, \quad(z, v) \mapsto v \lambda_{\operatorname{det}(v)}(z),
$$

является морфизмом, определяюшим действие тора $Z$ на множестве $\Omega$. Так как в $(34)$ мы имеем $\operatorname{det}(v) \neq 0$, то $\lambda_{\operatorname{det}(v)}(z)=v^{-1} \alpha((z, v))$, откуда следует, что отображение

$$
\beta: Z \times \Omega \longrightarrow \mathrm{SL}_{2}(k), \quad(z, v) \mapsto \lambda_{\operatorname{det}(v)}(z),
$$

является морфизмом.

Теперь рассмотрим действие группы $S$ на многообразии $Z \times \Omega$ через второй сомножитель. Мы имеем

$$
\pi_{Z \times \Omega, S}: Z \times \Omega \longrightarrow Z \times \mathbb{A}_{*}, \quad(z, v) \mapsto(z, \operatorname{det}(v)) .
$$

Из (35) и (36) следует, что морфизм $\beta$ постоянен на каждом слое морфизма $\pi_{Z \times \Omega, S}$. Поэтому ввиду определяюшего свойства категорного фактора сушествует такой морфизм $\gamma: Z \times \mathbb{A}_{*} \longrightarrow \mathrm{SL}_{2}(k)$, что $\beta=\gamma \circ \pi_{Z \times \Omega, S}$. С другой стороны, ясно, что $\beta=\lambda \circ \pi_{Z \times \Omega, S}$. Это показьвает, что $\lambda=\gamma$. Следовательно, $\lambda-$ морфизм.

6.10. Пусть $s$ - стандартная координатная функция на прямой $\mathbb{A}^{1}$, т.е. $s(c)=c$, $c \in \mathbb{A}^{1}$. Зафиксируем образующую $t$ групाы характеров тора $Z$. Тогда

$$
k\left[\mathbb{A}^{1}\right]=k[s], \quad k\left[\mathbb{A}_{*}\right]=k\left[s, s^{-1}\right], \quad k[Z]=k\left[t, t^{-1}\right] .
$$

Пусть $y_{1}$ и $y_{2}-$ переменные. Из (37) и предложения 3 следует, что сушествуют такие рациональные функции

$$
f_{i j}=f_{i j}\left(y_{1}, y_{2}\right) \in k\left[y_{1}, y_{1}^{-1}, y_{2}, y_{2}^{-1}\right], \quad 1 \leqslant i, j \leqslant 2,
$$

что в обозначениях (30) морфизм $\lambda$ определен формулой

$$
\lambda((z, c))=\left[f_{i j}(t(z), c)\right], \quad(z, c) \in Z \times \mathbb{A}_{*} .
$$

Теперь из (33), (34) и (39) следует, что морфизм $\alpha$ определен формулой

$$
\alpha((z, v))=\left[x_{i j}(v)\right]\left[f_{i j}(t(z), \operatorname{det}(v))\right], \quad(z, v) \in Z \times \Omega .
$$


6.11. Поскольку морфизм $\alpha$ является ограничением на множество $Z \times \Omega$ морфизма $Z \times V \longrightarrow V$, определяюшего действие тора $Z$ на пространстве $V$, а множество $Z \times \Omega$ открыто в многообразии $Z \times V$, из (40) следует, что все элементы матрицы $\left[x_{i j}\right]\left[f_{i j}(t, \operatorname{det})\right] \in \mathrm{SL}_{2}(k[Z \times \Omega])$ являются не просто рациональньми, а регулярными функциями на многообразии $Z \times V$, т.е.

$$
\begin{gathered}
x_{i 1} f_{1 j}(t, \text { det })+x_{i 2} f_{2 j}(t, \text { det }) \in k\left[t, t^{-1}, x_{11}, x_{12}, x_{21}, x_{22}\right]=k[Z \times V], \\
1 \leqslant i, j \leqslant 2 .
\end{gathered}
$$

ПРЕДЛОЖЕниЕ 4. $f_{i j} \in k\left[y_{1}, y_{1}^{-1}, y_{2}\right], \quad 1 \leqslant i, j \leqslant 2$.

ДокАЗАТЕЛЬСТво. Будем рассуждать от противного. Пусть, например, $f_{1 j} \notin$ $k\left[y_{1}, y_{1}^{-1}, y_{2}\right]$. Тогда ввиду $(38)$

$$
\begin{aligned}
& f_{1 j}(t, \operatorname{det})=\operatorname{det}^{n}\left(p_{0}+p_{1} \operatorname{det}+p_{2} \operatorname{det}^{2}+\cdots\right), \quad p_{i} \in k\left[t, t^{-1}\right], \quad p_{0} \neq 0, \\
& f_{2 j}(t, \operatorname{det})=\operatorname{det}^{l}\left(q_{0}+q_{1} \operatorname{det}+q_{2} \operatorname{det}^{2}+\cdots\right), \quad q_{i} \in k\left[t, t^{-1}\right], \quad q_{0} \neq 0,
\end{aligned}
$$

для некоторых целых чисел $n$ и $l$. Пусть $m=\min \{n, l\}$. Из (42) и (43) следует, что существует такой элемент $h \in k[Z \times V]$, что левая часть включения (41) равна

$$
f:=\operatorname{det}^{m}(r+h \operatorname{det}), \quad r=x_{i 1} p_{0} \operatorname{det}^{n-m}+x_{i 2} q_{0} \operatorname{det}^{l-m} .
$$

Поскольку числа $n-m$ и $l-m$ неотрищательны, то $r \in k[Z \times V]$. Так как хоть одно из них равно нулю, из (31) и (44) следует, что ограничение функции $r$ на множество $Z \times F_{0}$ отлично от нуля. Отсюда и из (44) вытекает, что функция $f$ имеет на множестве $Z \times F_{0}$ полюс. Это противоречит (41).

6.12. Итак, поскольку множество $Z \times \Omega$ открыто в многообразии $Z \times V$, мы установили, что действие тора $Z$ на пространстве $V$ задается формулой

$$
z \cdot v=v\left[f_{i j}(t(z), \operatorname{det}(v))\right], \quad z \in Z, \quad v \in V
$$

(по предложению 4 правая часть равенства (45) определена в любой точке $(z, v) \in$ $Z \times V)$.

6.13. Мы можем рассматривать отображение (33) как гомоморфизм групповых схем $\mathbb{G}_{m} \longrightarrow \mathrm{SL}_{2}$, определенный над кольцом $k\left[\mathbb{A}_{*}\right]=k\left[s, s^{-1}\right]$ (см. (37)). Поскольку тор $Z$ действует на пространстве $V$ с тривиальным ядром, этот гомоморфизм инъективен. Из (39) и предложения 4 следует, что в действительности он определен над кольцом $k\left[\mathbb{A}^{1}\right]=k[s]$. Воспользуемся теперь следуюшим утверждением.

Лемма 1. Пусть $A$ - факториальное кольцо и $\varphi: \mathbb{G}_{m} \longrightarrow \mathrm{SL}_{2}$ - инбективный гомоморфизм групповых схем, определенный над кольчом А. Тогда образ $T$ гомоморфизма $\varphi$ сопряжен с помощью әлемента из группы $\mathrm{SL}_{2}(A)$ диагональному тору. 
ДокАЗАТЕЛЬСТво. Пусть $x$ - образуюшая групшы характеров тора $\mathbb{G}_{m}$. Тогда гомоморфизм $\varphi$ задается матрицей $r=r(x) \in \mathrm{SL}_{2}\left(A\left[x, x^{-1}\right]\right)$, т.е. $\varphi(a)=r(x(a))$ для $a \in \mathbb{G}_{m}$.

Пусть $K$ - поле частных кольца $A$. Тогда $T$ является максимальным $K$-расщепимым тором в $\mathrm{SL}_{2}$. Следовательно [5], тор $T$ сопряжен с помощью элемента из $\mathrm{SL}_{2}(K)$ диагональному тору, т.е.

$$
r(t)=u \operatorname{diag}\left(t, t^{-1}\right) u^{-1}, \quad u=\left[u_{i j}\right] \in \mathrm{SL}_{2}(K) .
$$

Если $b$ - диагональная матрица из группы $\mathrm{SL}_{2}(K)$, то равенство (46) по-прежнему будет иметь место, если в его правой части заменить $u$ на $u b$. Отсюда вытекает, что можно считать, что

$$
u_{11} \quad \text { и } u_{21}-\text { взаимно простые элементы кольца } A .
$$

Поскольку правая часть равенства (46) равна

$$
\left[\begin{array}{ll}
u_{11} u_{22} t-u_{12} u_{21} t^{-1} & u_{11} u_{12} t^{-1}-u_{11} u_{12} t \\
u_{21} u_{22} t-u_{21} u_{22} t^{-1} & u_{11} u_{22} t^{-1}-u_{12} u_{21} t
\end{array}\right]
$$

условие $r \in \mathrm{SL}_{2}\left(A\left[t, t^{-1}\right]\right)$ показывает, что

$$
u_{11} u_{22}, \quad u_{12} u_{21}, \quad u_{11} u_{12}, \quad u_{21} u_{22} \in A
$$

Теперь из (47) и (48) легко вытекает, что $u \in \mathrm{SL}_{2}(A)$, откуда и следует утверждение леммы.

ЗАмЕчАниЕ 4. Групповую схему $\mathrm{SL}_{2}$ в формулировке леммы 1 нельзя заменить на групповую схему $\mathrm{SL}_{n}, n \geqslant 3[1]$.

6.14. Из леммы 1 следует, что сушествуют такие полиномы $u_{i j}=u_{i j}(y) \in k[y]$, $1 \leqslant i, j \leqslant 2$, где $y$-переменная, что $\left[u_{i j}(s)\right] \in \mathrm{SL}_{2}(k[s])$ и

$$
\left[u_{i j}(s)\right]\left[f_{i j}(t, s)\right]\left[u_{i j}(s)\right]^{-1}=\operatorname{diag}\left(t, t^{-1}\right)
$$

Пусть $\sigma$ - автоморфизм многообразия $V$, определенный формулой

$$
\sigma: V \longrightarrow V, \quad v \mapsto v\left[u_{i j}(\operatorname{det}(v))\right]
$$

Мы имеем $\sigma^{-1}(v)=v\left[u_{i j}(\operatorname{det}(v))\right]^{-1}$. Поэтому из (45) и (50) следует, что для любого элемента $z \in Z$, рассмотренного как автоморфизм многообразия $V$, имеем

$$
\begin{gathered}
\sigma^{-1} \circ z \circ \sigma: V \longrightarrow V, \\
v \mapsto v\left[u_{i j}(\operatorname{det}(v))\right]\left[f_{i j}\left(t(z), \operatorname{det}\left(v^{\prime}\right)\right)\right]\left[u_{i j}\left(\operatorname{det}\left(v^{\prime \prime}\right)\right)\right]^{-1},
\end{gathered}
$$


где $v^{\prime}=v\left[u_{i j}(\operatorname{det}(v))\right], v^{\prime \prime}=v^{\prime}\left[u_{i j}\left(\operatorname{det}\left(v^{\prime}\right)\right)\right]$.

Поскольку матрица $\left[u_{i j}(s)\right]$ унимодулярна, $\operatorname{det}\left(v^{\prime \prime}\right)=\operatorname{det}\left(v^{\prime}\right)=\operatorname{det}(v)$. Следовательно, согласно (51) и (49) для любого элемента $z \in Z$ мы имеем

$$
\sigma^{-1} \circ z \circ \sigma: V \longrightarrow V, \quad v \mapsto v \operatorname{diag}\left(t(z), t^{-1}(z)\right)
$$

Формула (52) показывает, что $\sigma^{-1} \circ z \circ \sigma-$ линейный автоморфизм пространства $V$. Поскольку автоморфизм $\sigma$ является $S$-эквивариантным, мы заключаем отсюда, что $\sigma^{-1} \circ g \circ \sigma$ является линейным автоморфизмом пространства $V$ для любого элемента $g \in G$.

Этим завершается доказательство линеаризуемости в рассматриваемом случае, а вместе с ним - и доказательство теоремы 2.

\section{Список литературы}

1. Bass H. Письмо к автору (апрель 11, 1997).

2. Bass H., Haboush W. Linearizing certain reductive group actions // Trans. Amer. Math. Soc. 1985. V. 292. № 2. P. 463-482.

3. Biatynicki-Birula A. Remarks on the action of an algebraic torus on $k^{n}$. I // Bull. Acad. Polon. Sci. Ser. Sci. Math., Astr., Phys. 1967. V. XIV. P. 177-188.

4. Biatynicki-Birula A. Remarks on the action of an algebraic torus on $k^{n}$. II // Bull. Acad. Polon. Sci. Ser. Sci. Math., Astr., Phys. 1967. V. XV. P. 123-125.

5. Борель А. Линейные алгебраические группы. М.: Мир, 1972.

6. Элашвили А. Г. Канонический види стационарные подалгебры точек общего положения для простых линейных групп Ли // Функц. анализ и его прилож. 1972. Т. 6. С. 51-62.

7. Элашвили А.Г. Стационарные подалгебры точек общего положения для неприводимых линейных групп Ли // Функц. анализ и его прилож. 1972. Т. 6. С. 65-78.

8. Горбащевич В. В., Онищик А. Л. Группы Ли преобразований // Современные проблемы математики. Фундаментальные направления. Т. 20. М.: ВИНИТИ, 1988. С. 103-240.

9. Kaliman S., Makar-Limanov L. On the Russell-Koras contactible 3-folds // J. Alg. Geom. 1997. V. 6. P. 247-268.

10. Kaliman S., Makar-Limanov L., Koras M., Russell P. $\mathbb{C}^{*}$-actions on $\mathbb{C}^{3}$ are linearizable // E. Res. Announc. 1997. V. 3. P. 63-71.

11. Kambayashi T. Automorphism group of a polynomial ring and algebraic group action on an affine space // J. of Algebra. 1979. V. 60. P. 439-451.

12. Kambayashi T., Russell P. On linearizing algebraic torus actions // J. Pure Appl. Algebra. 1982. V. 23. P. $243-250$.

13. Kimura T. A classification of prehomogeneous vector spaces of simple algebraic groups with scalar multiplications // J. Algebra. 1983. V. 83. № 1. P. 72-100.

14. Koras $M$. A characterization of $\mathbb{C}^{2} / \mathbb{Z}_{a} / /$ Comp. Math. 1993. V. 87. P. 241-267.

15. Koras M., Russell P. $\mathbb{G}_{m}$-actions on $\mathbb{A}^{3} / /$ Can. Math. Soc. Conf. Proc. 1989. V. 10. P. 269-276.

16. Koras M., Russell $P$. $\mathbb{C}^{*}$-actions on $\mathbb{C}^{3}$ : the smooth locus is not of hyperbolic type // CICMA reports. 1996. V. 06.

17. Koras M., Russell P. Contractible 3-folds and $\mathbb{C}^{*}$-actions on $\mathbb{C}^{3} / /$ J. Alg. Geom. 1997. V. 6. P. 671-695.

18. Kraft $H$. Challenging problems on affine $n$-space // Séminaire Bourbaki, 47ème année. 1994-95. V. 802. P. 1-19.

19. Kraft H., Popov V.L. Semisimple group actions on the three-dimensional affine space are linear // Comment. Math. Helvetici. 1985. V. 60. P. 466-479. 
20. Kurth A. Nonlinear equivariant automorphisms // Manuscr. Math. 1997. V. 94. P. 327-335.

21. Luna D. Slices étales // Bull. Soc. Math. France. 1973. V. 33. P. 81-105.

22. Masuda M., Petrie T. Algebraic families of $O(2)$-actions on affine space $\mathbb{C}^{4} / /$ Proc. Symp. Pure Math. 1994. V. 54. № I. P. 347-354.

23. Винберг Э. Б., Горбачевич В. В., Онищик А. Л. Строение групп и алгебр Ли // Современные проблемы математики. Фундаментальные направления. Т. 41. М.: ВИНИТИ, 1990.

24. Панюшев Д. И. Полупростые группы автоморфизмов четырехмерного аффиинного пространства // Изв. АН СССР. Сер. матем. 1983. Т. 47. № 4. С. 881-894.

25. Попов В. Л. Критерий стабильности действия полупростой группы на факториальном многообразии // Изв. АН СССР. Сер. матем. 1970. Т. 34. № 3. С. 523-531.

26. Попов В. Л. Классификация трехмерных аффинных алгебраических многообразий, квазиоднородных относительно алгебраической группы // Изв. АН СССР. Сер. матем. 1975. T. 39. № 3. C. 566-609.

27. Popov V.L. Algebraic actions of connected reductive algebraic groups on $\mathbb{A}^{3}$ are linearizable // Preprint. June 20, 1996.

28. Popov V.L. Polynomial automorphisms of affine spaces: connected reductive subgroups of Aut $\mathbb{A}^{3}$ and Aut $\mathbb{A}^{4} / /$ Preprint. December 25, 1997.

29. Винберг Э. Б., Попов В. Л. Теория инвариантов // Современные проблемы математики. Фундаментальные направления. Т. 55. М.: ВИНИТИ, 1989. С. 137-314.

30. Rosenlicht M. Toroidal algebraic groups // Proc. Amer. Math. Soc. 1961. V. 12. P. 984-988.

31. Sato M., Kimura T. A classification of irreducible prehomogeneous vector spaces and their relative invariants // Nagoya Math. J. 1977. V. 65. P. 1-155.

32. Shafarevich I. R. On some infinite dimensional algebraic groups // Rend. Math. e Appl. 1966. V. 25. № 2. P. 208-212.

Московский государственный институт

Поступило в редакцию

электроники и математики,

6.III. 2000

Большой Трехсвятительский пер., 3/12,

Москва, 109028, Россия

E-mail: popov@ppc.msk.ru 